\title{
A 55 Year Old Man with Progressive Neurologic Deficits
}

\author{
Hardy Senjaya \\ Department of Anatomy, Medical Faculty, Trisakti University, Jakarta 11440, Indonesia
}

\begin{abstract}
At least $50 \%$ of the decline in functional abilities associated with the elderly is caused by neurological conditions, particularly vascular dementia, such as occurs in Binswanger disease. Binswanger disease is a rare condition, characterized by acute strokes with symptoms and signs compatible with lacunar infarction. The disease onset is commonly between 55 and 75 years. The majority of patients with Binswanger disease have chronic hypertension and other putative factors including diabetes mellitus, polycythemia, thrombocytosis, hyperlipidemia, hyperglobulinemia and pseudoxanthoma elasticum, increased fibrinogen levels and the antiphospholipid antibody syndrome. We report the case of a 55 year old man, who suffered from involuntary movements of his right arm, slight hemiparesis on the right side, and also had dementia. He had a history of high blood pressure and laboratory tests showed that he had diabetes mellitus. The brain magnetic resonance imaging showed irregular white matter abnormalities with multiple lacunar infarcts in the basal ganglia and pons. The clinical picture is characterized by acute strokes, followed by involuntary movements and also dementia. Therefore we decided to diagnose it as Binswanger disease. After discharge from hospital, the patient has not returned for follow-up.
\end{abstract}

Key words: Binswanger disease, irregular white matter abnormalities, multiple lacunar infarcts.

\section{Introduction}

Aging is associated with a decline in functional abilities and neurologic conditions are responsible for at least $50 \%$ of this decline. Neurologic conditions especially vascular dementia and stroke are the leading causes of disability and institutionalization in the elderly. A distinctive form of small vessel disease causing dementia is ischemic white matter disease or Binswanger's disease.

The concept that dementia is a group of cognitive disorders with many underlying subtypes and pathophysiological causes was first defined by Otto Binswanger, Alois Alzheimer, and Emil Kraepelin at the close of the 19th century. However, at the end of the 20th century, the descriptive criteria, incidence, and prevalence for many of these dementias are still subject to controversy [1]. One subtype, labeled Binswanger's disease by Alzheimer in 1902, referred to arteriosclerotic subcortical white matter changes [2].

\footnotetext{
Corresponding author: Hardy Senjaya, M.D., Ph.D., research field: clinical anatomy. E-mail: hardysenjaya49@gmail.com.
}

BD (Binswanger's disease), one of the most common forms of vascular dementia in the elderly, is also called subcortical vascular dementia, or subacute arteriosclerotic encephalopathy. The clinical features of $\mathrm{BD}$ are highly variable and the psychiatric and behavioral manifestations have been less well described. Patients with BD have dementia and various clinical and radiological abnormalities, such as focal cerebrovascular deficits, and subcortical cerebral dysfunction. They also have a variety of vascular risk factors [3-5].

Pathologically there are widespread, microscopic areas of damage to the deep layers of white matter in the brain. The disorder is characterized by prominent brain atrophy with ventricular dilation, diffuse demyelination of the white matter, and a scattering of lacunar infarcts in the basal ganglia and white matter. The brain damage is the result of thickening and narrowing (atherosclerosis) of arteries that feed the subcortical areas of the brain [6].

Atherosclerosis (popularly known as "hardening of the arteries”) is a systemic process that affects blood vessels throughout the body. It begins late in the 
fourth decade of life and increases in severity with age [6]. Cerebral blood vessels undergo profound changes with aging. In the cerebral cortex and hippocampus cerebral capillaries are reduced in number and have thickened and fibrotic basement membranes [7]. These alterations reduce cerebral perfusion, deplete cerebrovascular reserves, and increase the susceptibility of the brain to vascular insufficiency and ischemic injury [8].

Chronic hypertension is associated with profound changes in the structure and function of cerebral blood vessels. Hypertension progressive neurologic deficits promote atherosclerosis in cerebral arteries [9] and induce lipohyalinosis, a pathological process characterized by fibrinoid necrosis of the vascular wall [9]. Functional changes include regionally reduced resting CBF (cerebral blood flow) and impaired cerebrovascular reactivity [10]. Therefore functional hyperemia is attenuated in hypertensive patients. The following is a case report of Binswanger's disease as encountered by the authors.

\section{Case Report}

A 55-year old man who came from a remote area, was admitted to our clinic in July 2002. He complained of involuntary swinging movements of his right arm. Before visiting us, this patient had been treated by the neurologist of the local hospital and was diagnosed as having CVD (cardiovascular disease) ischemic stroke. Two weeks later when this patient was treated in the same hospital, the patient's family noticed that his memory became worse and also that he appeared to have difficulty in moving his limbs.

He had a history of hypertension, and took anti-hypertensive drugs irregularly. He did not know whether he had diabetes mellitus or not. On physical examination in our clinic at the time of admission the patient had a good general performance and was fully alert with blood pressure 170/100 mm Hg, and pulse $92 \mathrm{x} / \mathrm{min}$. There were no signs of meningeal iritation and no papilledema on bilateral funduscopy. The pupils were of equal size and of normal shape, light reflex $+/+$. There was slight right hemiparesis and occasionally involuntary movements of the right arm were seen. Physiologic reflexes were normal and there were no pathological reflexes. At the time of admission our working diagnosis was CVD ischemic stroke. We observed this patient as an inpatient and he underwent several diagnostic tests as shown in Table 1.

Table 1 Laboratory data of haematologic and lipid profile.

\begin{tabular}{ll}
\hline Laboratory parameters & \\
\hline Hb (haemoglobin) & $15.7 \mathrm{~g} / \mathrm{dL}$ \\
WBC (white blood cell) & $9.96 \mathrm{Th} / \mathrm{UI}$ \\
Different count: Basophile/Eosinophile/Stab cell/Neotrophile/Lymphocytes/Monocyte & $0 / 4 \% / 0 / 60 \% / 30 \% / 6 \%$ \\
Platelets & $254.000 \mathrm{Th} / \mathrm{UI}$ \\
ESR (erythrocyte sedimentation rate) & $15 \mathrm{~mm} / \mathrm{hour}$ \\
Blood urea nitrogen & $13 \mathrm{mg} / \mathrm{dL}$ \\
Creatinine & $1.0 \mathrm{mg} / \mathrm{dL}$ \\
Uric acid & $7 \mathrm{mg} / \mathrm{dL}$ \\
Blood glucose, fasting & $189 \mathrm{mg} / \mathrm{dL}$ \\
Blood glucose, 2 hours after meal & $213 \mathrm{mg} / \mathrm{dL}$ \\
Total cholesterol & $200 \mathrm{mg} / \mathrm{dL}$ \\
High density lipoprotein & $45 \mathrm{mg} / \mathrm{dL}$ \\
Low density lipoprotein & $128 \mathrm{mg} / \mathrm{dL}$ \\
Triglycerides & $135 \mathrm{mg} / \mathrm{dL}$ \\
Lipoprotein a & $7.0 \mathrm{mg} / \mathrm{dL}$ \\
Homocysteine & $9.2 \mathrm{umol} / \mathrm{L}$ \\
Fibrinogen (patient) & $240 \mathrm{mg} / \mathrm{dL}$ \\
Fibrinogen (control) & $326 \mathrm{mg} / \mathrm{dL}$ \\
Platelet agregation & Normoagregation \\
\hline
\end{tabular}


Echocardiography: Valvular heart disease (mild-moderate aortic regurgitation). TCCD (Transcranial color doppler sonography): All the cerebral circulation (anterior and posterior) showed low doppler velocity generalized cerebral atherosclerosis. Chest X-ray: Normal chest. Brain magnetic resonance $300 \mathrm{mg}$ in $500 \mathrm{~mL}$ asering per 12 $\mathrm{hr}$, piracetam injection $3 \mathrm{~g}$ four times daily, cilostazol (oral) $50 \mathrm{mg}$ twice daily, donepezil $5 \mathrm{mg}$ once daily, quinapril HCL (Accupril) $10 \mathrm{mg}$ once daily, glipizide $5 \mathrm{mg}$ once daily. After discharge from hospital, the patient has not returned for follow-up.

\section{Results and Discussion}

Binswanger's disease (encephalopathy or leucoaraiosis) is a rare disorder of presumed vascular etiology, characterized by progressive dementia and pseudobulbar palsy. The histopathology shows thinning and hyalinization of the walls of small penetrating arteries and occasionally amyloid angiopathy in the basal ganglia and periventricular regions. Associated with diffuse hemispheric demyelination resulting from chronic ischemic disease, there is axonal loss and gliosis, probably due to anoxia as a result of sclerotic changes [11].

The age of onset of senile dementia of the Binswanger's type is typically in the sixth and seventh decades of life, and men and women appear to be affected equally often. The ncidence of the disease is difficult to cite progressive neurologic deficits given the controversies regarding its clinical features but has been reported to range from $3 \%$ to $12 \%$ in the elderly [12].

This patient came to our clinic from a remote area with swinging involuntary movements of his right arm. Before he visited us, he had been treated at the local hospital as an ischemic stroke patient. When the patient was under treatment, his family noticed that his memory became worse and that he appeared to have difficulty in moving his limbs.

The brain MRI showed irregular white matter abnormalities and multiple lacunar infarcts in the basal ganglia and also in the pons (Figs. 1 and 2). There was no evidence of hydrocephalus (Fig. 3). This patient had hypertension and diabetes mellitus and TCCD examination indicated the impression of generalized cerebral atherosclerosis.

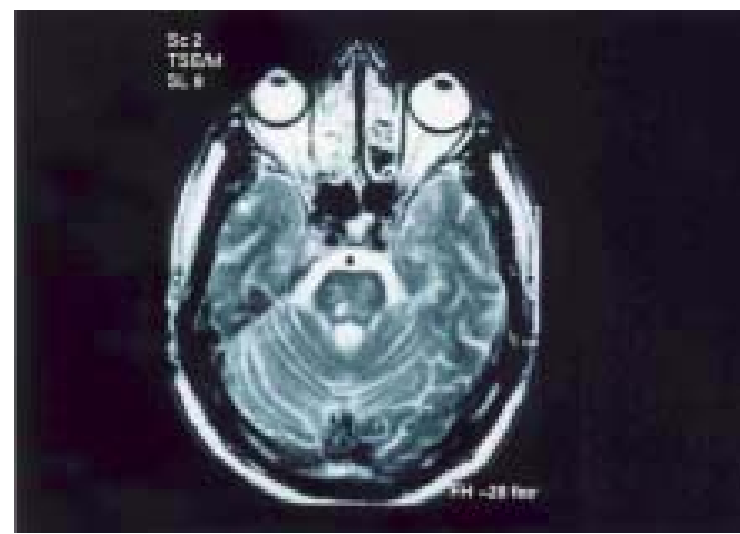

Fig. 1 T2 weighted MRI showing.

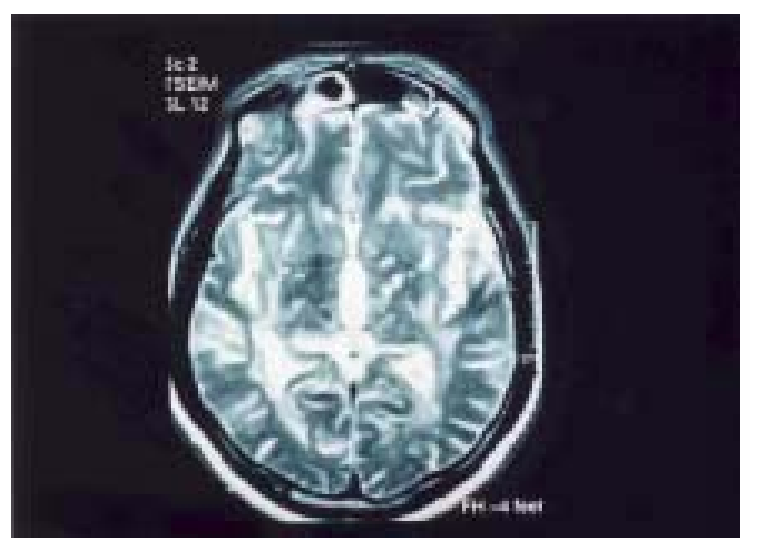

Fig. 2 T2 irregular in pons weighted MRI showing irregular white matter abnormalities and multiple lacunar infarctions in basal ganglia.

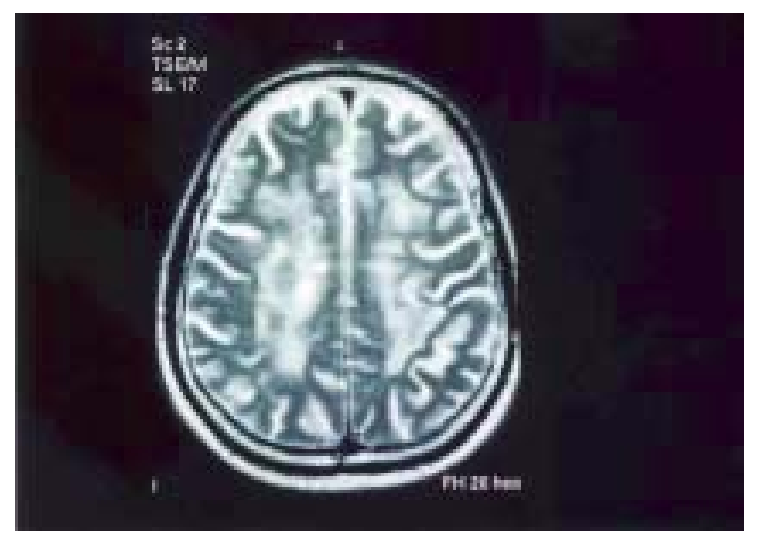

Fig. 3 T2-weighted MRI showing no evidence of hydrocephalus. 
The pathogenesis of $\mathrm{BD}$ and the relation between $\mathrm{BD}$ and the multiple lacunar infarctions that accompany the white matter lesions remain unclear. However, it is likely that chronic cerebral ischemia is common to these two conditions. In addition, it has been suggested that these white matter lesions may be caused by arterial hypertension and a subsequent dysfunction of the blood-brain barrier. Lipohyalinosis (arteriolosclerosis), induced by chronic hypertension or diabetes or both, is the most common finding in the small vessels of deep brain from patients with white matter disease [13].

BD occurs equally in both sexes with onset between 55 and 75 years. The clinical picture is characterized by acute strokes with symptoms and signs compatible with lacunar infarction. Subacute onset of focal neurological deficits may develop over a period of several days, and there is a stepwise progression of motor, cognitive, intellectual, and behavioral deficits during a 5-10 year period. Corticospinal and extrapyramidal signs may also occur.

The majority of patients with $\mathrm{BD}$ have chronic hypertension that could cause degenerative changes in the penetrating arteries of the white matter. Other putative factors include diabetes mellitus, polycythemia, thrombocytosis, hyperlipidemia, hyperglobulinemia, and pseudoxanthoma elasticum, increased fibrinogen levels and increased serum viscosity antiphospholipid antibody syndrome.

This patient had the clinical picture of BD, such as acute stroke, extrapyramidal signs (involuntary movements), and dementia. He also had a history of hypertension and diabetes mellitus.

After complete diagnostic examinations we decided to establish the diagnosis of Binswanger's disease in this patient. Although we could not find the other putative factors such as polycythemia, thrombocytosis, increased fibrinogen levels and others, all his symptoms (high blood pressure, diabetes mellitus and more importantly, the brain MRI) were typical of Binswanger disease.
$\mathrm{BD}$ is a progressive disease for which there is no cure. Changes may be sudden or gradual and then progress in a stepwise manner. BD can often coexist with Alzheimer's disease. If progression of BD can be halted or slowed with treatments then there is that much more time that the patient can properly function and retain his or her personality. Until more studies can be done, however, all this is speculation and BD is still a sentence of life-imprisonment and eventual death to the body [14].

The victims of Binswanger's disease have gradually progressive aspontaneity, slowed cognition, frequent falls, upper motor neuron signs, and possibly seizures. In the future, newer laboratory findings may support the diagnosis of BD during life, such as magnetization transfer images [15], ischemia on diffusion-weighted MRI [16], slowed arterial-venous transit times by transcranial sonography [17] and decreased cerebral reactivity to apnea during cognitive tasks [18].

\section{Conclusions}

This patient was unique in having extreme BD without accompany lacunae or cystic lessions, as are usually seen in the presence of hypertensive cerebrovascular arteriolosclerosis.

\section{Acknowledgements}

We wish thank to Senjaya Muljadi M.D., specialist in neurology as consultant.

\section{References}

[1] Roman, G. C. 1999. “A Historical Review of the Concept of Vascular Dementia: Lessons from the Past for the Future.” Alzheimer Dis. Assoc. Disord. 13 (suppl. 3): S4-S8.

[2] Hurley, R. A., Tomimoto, H., Akiguchi, I., Fisher, R. E., and Taber, K. H. 2000. "Binswanger's Disease: An Ongoing Controversy.” J. Neuropsychiatry Clin. Neurosci. 12: 301-4.

[3] Tomimoto, H., Akiguchi, I., Wakita, H., Osaki, A., Hayashi, M. and Yamamoto, Y. 1999. "Coagulation Activation in Patients with Binswanger Disease.” Arch. Neurol. 56: 1104-8. 
[4] Román, G. C. 1999. "New Insight into Binswanger Disease.” Arch. Neurol. 56: 1061-2.

[5] Nagaratnam, N. and Nagaratnam, K. 1998. "Psychiatric and Behavioral Aspects of Dementia of the Binswanger Type.” Am. J. Alzheimer's Dis. Other Demen. 13: 173-8.

[6] National Institute of Neurological Disorders and Stroke. 2009. "NINDS Binswanger's Disease Information Page." Accessed July 1, 2009.

http://www.ninds.nih.gov/disorders/binswangers/binswan gers.htm.

[7] Farkas, E. and Luiten, P. G. 2001. “Cerebral Microvascular Pathology in Aging and Alzheimer's Disease.” Prog. Neurobiol. 64: 575-611.

[8] Fernando, M. S., Simpson, J. E., Matthews, F., Brayne, C., Lewis, C. E., Barber, R., Kalaria, R. N., Forster, G., Esteves, F., Wharton, S. B., Shaw, P. J., O'Brien, J. T., Ince, P. G., and MRC Cognitive Function and Ageing Neuropathology Study Group. 2006. "White Matter Lesions in an Unselected Cohort of the Elderly: Molecular Pathology Suggests Origin from Chronic Hypoperfusion Injury.” Stroke 37: 1391-8.

[9] Droste, D. W., Ritter, M. A., Dittrich, R., Heidenreich, S., Wichter, T., Freund, M., and Bernd Ringelstein, E. 2003. "Arterial Hypertension and Ischaemic Stroke." Acta Neurol. Scand. 107: 241-51.

[10] Iadecola, C., and Davisson, R. L. 2008. "Hypertension and Cerebrovascular Dysfunction.” Cell Metab. 7:476-84.

[11] Gilroy, J. 2000. "Binswanger Disease." In Basic Neurology. 3rd ed. USA, New York: The McGraw Hill Companies, Inc., 261-2.
[12] Olsen, C. G., and Clasen, M. E. 2009. Senile Dementia of the Binswanger's Type. American Academy of Family Physicians. Accessed July 1, 2009. http://www.aafp.org/afp/AFPprinter/981200ap/olsen.html ?print=yes.

[13] Palumbo, V., Boulanger, J. M., Hill, M. D., Inzitri, D., and Buchan, A. M. 2007. "Leukoaraiosis and Intracerebral Hemorrhage after Thrombolysis in Acute Stroke.” Neurology 68: 1020-4.

[14] Kohshiro, F., Sadaharu, T., Hiroaki, H., and Mitsuko, S. 2005. "Binswanger's Disease: Clinical and Computed Tomograph Neuroradiological Study of Seven Cases.” Psychogeriatrics 5: 127-46.

[15] Hanyu, H., Asano, T., Sakurai, H., Iwamoto, T., Takasaki, M., Shindo, H., and Abe, K. 1999. "Magnetization Transfer Ratio in Cerebral White Matter Lesions of Binswanger's Disease.” J. Neurol. Sci. 166: 85-90.

[16] Choi, S. H., Na, D. L., and Chung, C. S. 2000. "Diffusion-Weighted MRI in Vascular Dementia." Neurology 54: 83-9.

[17] Puls, I., Hauck, K., Demuth, K., Horowski, A., Schlieber, M., Dörfler, P., Scheel, P., Toyka, K. V., Reiners, K., Schöning, M., and Becker, G. 1999. "Diagnostic Impact of Cerebral Transit Time in the Identification of Microangiopathy in Dementia: A Transcranial Ultrasound Study.” Stroke 30: 2291-5.

[18] Lim, G. T., Mendez, M. F., Bronstein, Y. L., Jouben-Steele, L. and Vinters, H. V. 2002. "Clinicopathologic Case Report: Akinetic Mutism with Findings of White Matter Hyperintensity." $J$. Neuropsychiatry Clin. Neurosci. 14: 214-21. 